\title{
Solving the Pose Ambiguity via a Simple Concentric Circle Constraint
}

\author{
Mingliang $\mathrm{Fu}^{1}$ and Weijia Zhou ${ }^{2}$ \\ ${ }^{1}$ Student, State Key Laboratory of Robotics, Shenyang Institute of Automation, Chinese Academy of Sciences, Institutes for \\ Robotics and Intelligent Manufacturing, Chinese Academy of Sciences, University of Chinese Academy of Sciences, CHINA \\ ${ }^{2}$ Professor, State Key Laboratory of Robotics, Shenyang Institute of Automation, Chinese Academy of Sciences, Institutes for \\ Robotics and Intelligent Manufacturing, Chinese Academy of Sciences, CHINA \\ ${ }^{1}$ Correspondence Author: fumingliang@ @ia.cn

\begin{abstract}
Estimating the pose of objects with circle feature from images is a basic and important question in computer vision community. This paper is focused on the ambiguity problem in pose estimation of circle feature, and a new method is proposed based on the concentric circle constraint. The pose of a single circle feature, in general, can be determined from its projection in the image plane with a pre-calibrated camera. However, there are generally two possible sets of pose parameters. By introducing the concentric circle constraint, interference from the false solution can be excluded. On the basis of element at infinity in projective geometry and the Euclidean distance invariant, cases that concentric circles are coplanar and non-coplanar are discussed respectively. Experiments on these two cases are performed to validate the proposed method.
\end{abstract}

Keywords-- Monocular Vision, Pose Estimation, Ambiguity, Concentric Circle

\section{INTRODUCTION}

The circular feature is basic geometric shape of objects around us. Many researchers [1-6] choose circular patterns to calibrate camera parameters. Also, it is used as a target feature to localize and recognize objects in robotic vision. Therefore, getting the position and orientation of a circle feature from 2D images with respect to a reference frame is of fundamental importance.

It is generally known that there are generally two possible 3D circles in different poses of which projections are the same. Safaee-Rad et al.[7] gave a closed-formed analytical solution to the problem of 3D pose estimation of circular features. Shiu et al. [8] presented another method to obtain the closed form solution for this problem on the basis of analytical geometry. Chen et al.[9] described a circle feature based pose estimation method which is directly based on two particular chords of the circular object. The corresponding 2-D feature chords can be defined by using two particular planes related to the $3-\mathrm{D}$ viewing cone constructed from the image of the circular object. Zheng et al.[10] employed the projective equation of a circle and presented a brand new geometric explanation for the ambiguity problem. Unfortunately, these researchers[7-10] did not give a program to eliminate the false solution. Additional information of a laser ranger finder[11] was used to select the correct one from two possible solutions. An auxiliary coplanar point and skew lines were introduced respectively as additional constraints to differentiate the real pose from the other[12,13].
This study presents a method to determine the correct pose using a concentric circle constraint. When two concentric circles are coplanar, the imaged circular points (ICPs) can be uniquely determined[14]. Thus, the false solution can be excluded immediately. In the case of noncoplanar situation, the false solution can be removed with the Euclidean distance invariant. Detailed proofs are given and experiment results show that the proposed method is valid. The original conference version of this paper can be found in [15]. More results in non-coplanar cases are included in this version.

The remaining part of this paper is organized as follows. Section 2 presents the pinhole camera model and the pole-polar relationship in projective geometry. The ambiguity in pose estimation of single circle feature and details of our method using the concentric circle constraint are elaborated in Section 3. Test results are presented in Section 4. Finally, conclusions are given in Section 5.

\section{BASIC CONCEPTS AND PRINCIPALS}

Without loss of generality, the following discussions are based on the prerequisite that the camera used in our experiments is pre-calibrated. In order to keep units the same, all the image coordinates are transformed to the normalized focus plane of which focus length is 1 . So the results are in the focal length $f$.

\subsection{THE CAMERA MODEL}

As it can be seen in the previous publications $[10,11]$, a general projection camera can be modeled as 
ideal pinhole imaging model.A 3D point $x \in \square^{3}$ in space is

mapping

projected onto an image point $m \in \square^{2}$ according to the

$$
s \tilde{m}=P \tilde{x}=K[R, t] \tilde{x}=K\left[r_{1}, r_{2}, r_{3}, t\right] \tilde{x}
$$

where $s$ is a scale factor, $P$ is the projection matrix, $R$ and $t$ represent the relative rotation and translation between the world reference frame and the camera coordinate frame, $\tilde{m}$ and $\tilde{x}$ are the homogeneous coordinates of image point $m$ and 3D point $x$, respectively.

\subsection{THE POLE-POLAR RELATIONSHIP}

A point $x$ and conic $C$ define a line $l=C x$. The line $l$ is called the polar of $x$ with respect to $C$, and the point $x$ is the pole of $l$ with respect to $C$.The polar line $l$ intersects the conic $C$ in two points. The two lines are tangent to $C$ at these points intersect at the point $x$.The above relationships are illustrated in Fig. 1. Specifically, suppose the conic $C$ is the projection of a 3D circular feature and $x$ is the image of the circle center. Thus the line determined from the conic $C$ and the center image $x$ is the vanishing line[16].

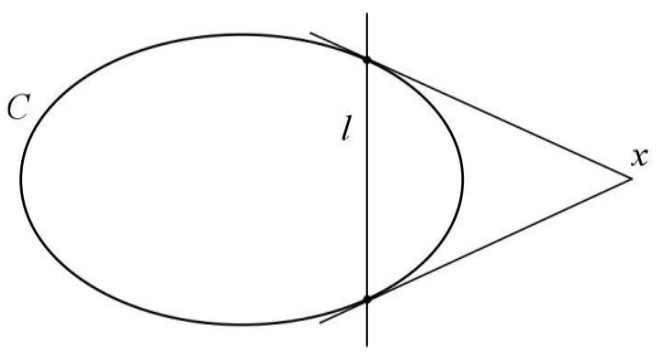

Figure 1: The pole-polar relationship.

\section{METHOD FOR THE SOLUTION OF AMBIGUITY IN POSE ESTIMATION}

To find 3D positions of circular features with monocular monocular vision system, this study proposed to employ the concentric circle constraint to remove the false pose. Cases that concentric circles are coplanar and noncoplanar are discussed in this section, respectively. Next, this study presents a description of the pose ambiguity.

\subsection{THE AMBIGUITY PROBLEM IN POSE ESTIMATION OF A CIRCULAR FEATURE}

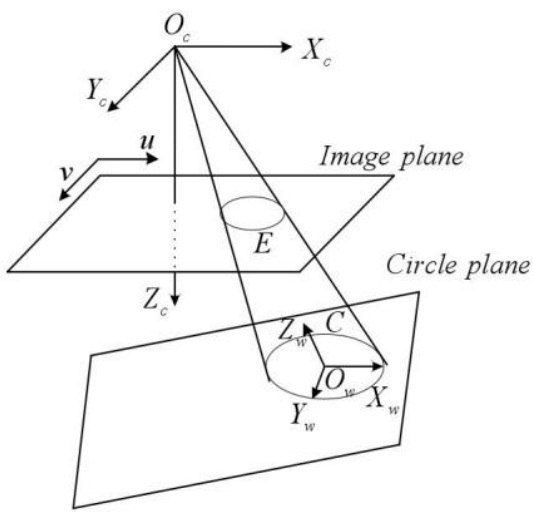

Figure 2: The pinhole camera model in the scene.

The position and orientation of a circular feature in 3D space is completely specified by the coordinates of its center and the normal vector. Here this study introduces a novel explanation [10] for the ambiguity based on the ICPs. Three coordinate frames are shown in Fig. 2. The origin of the world coordinate system (WCS) is at the center of the 
circle $C$, and the circle $C$ is in the plane $X_{w} O Y_{w}$. The camera coordinate system (CCS) $O_{c}-X_{c} Y_{c} Z_{c}$ is a three dimensional frame with the origin $O_{c}$, and the coordinate axis $Z_{c}$ is perpendicular to the image plane. The image $Q=\operatorname{diag}\left\{1,1,-r^{2}\right\}(2)$

And its dual form can be expressed as:

$$
Q_{\text {dual }}=Q^{-1}=\operatorname{diag}\left\{1,1,-1 / r^{2}\right\} \text { (3) }
$$

With the projection matrix $P$ inthe equation (1), the dual circle image $C^{*}$ satisfies:

$$
\begin{aligned}
& k C^{*}=P Q_{\text {dual }} P^{T} \\
& =K\left[\begin{array}{lll}
r_{1} & r_{2} & T
\end{array}\right] \operatorname{diag}\left\{\begin{array}{lll}
1 & 1 & -1 / r^{2}
\end{array}\right\}\left[\begin{array}{lll}
r_{1} & r_{2} & T
\end{array}\right]^{T} K^{T} \\
& =K K^{T}-\left(K r_{3}\right)\left(K r_{3}\right)^{T}-1 / r^{2}(K T)(K T)^{T}
\end{aligned}
$$

where $v=K r_{3}$ is the vanishing point of the circle's normal, $q=(K T) / r$ is the image coordinates of the

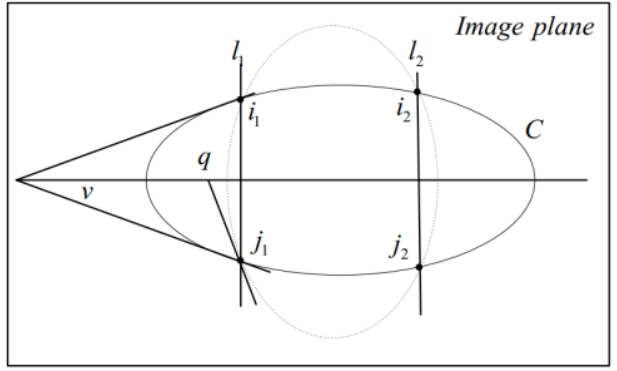

(a) frame $u-v$ is a $2 \mathrm{D}$ frame of which the axis $u$ and $v$ are parallel to the axis $X_{c}$ and $Y_{c}$ of CCS, respectively.

As shown in Fig. 2, let $r$ denotes the 3D circle radius. The circular feature $C$ in WCS can be written in a matrix form as circle's center, $\omega^{*}=K K^{T}$ is the inverse of the image of the absolute conic (IAC).

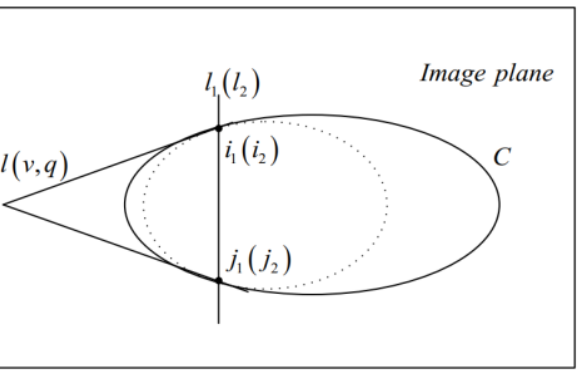

(b)

Figure 3: Projections of the absolute conic and the circle: (a) the general case and (b) a special case.

The relationship between the IAC and the image of projected circle is shown in Fig.3. The image of the circle $C$ intersects the IAC at four complex points, which can be divided into two pairs of complex conjugate points. Only two of them are the real ICPs [10].

3.2 COPLANAR CONCENTRIC CIRCLES
In the case of coplanar concentric circles, the projection of two concentric circles $C_{1}$ and $C_{2}$ in 3D space takes the form of ellipse [8] (except in degenerated cases where the projection is a segment), which can be represented as two ellipses $e_{1}$ and $e_{2}$. As depicted in Fig.4(a), two viewing cone are defined by the same base (the supporting plane of two concentric circles) and a vertex (the center of the camera's lens). 


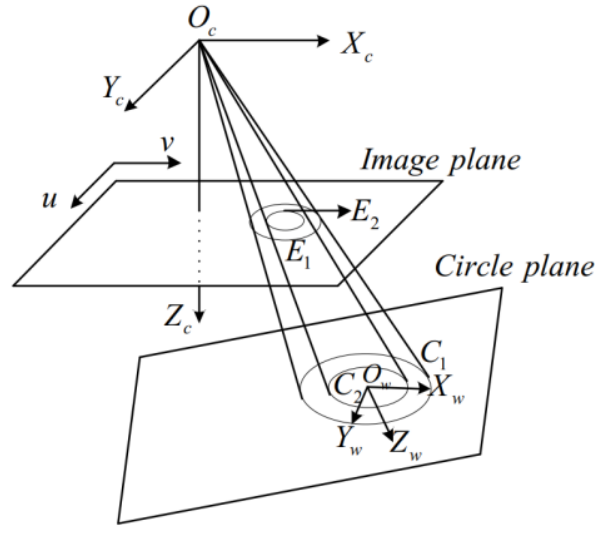

(a)

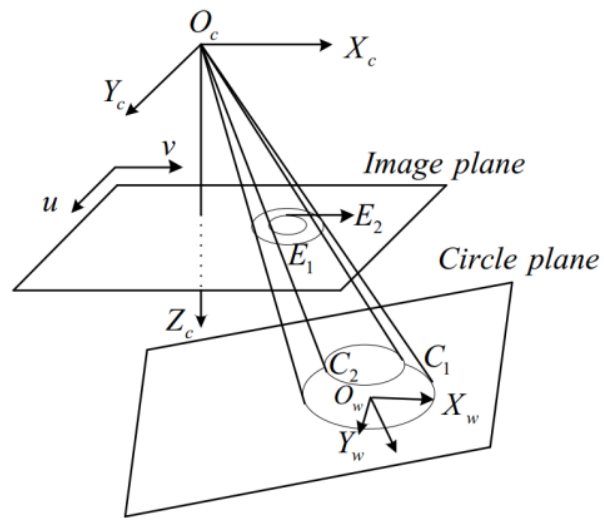

(b)

Figure 4: Projections of the concentric circles: (a) the coplanar case and (b) the non-coplanar case.

Proposition1. Suppose $\mathrm{o}_{c}$ is the image of the concentric circle center, $\omega_{c 1}$ is the ellipse equation which represents the image of the circle $C_{1}$ in $3 \mathrm{D}$ space. Then the vanishing line $l_{\infty}$ can be determined by a pole-polar relationship between the center image $\mathrm{o}_{c}$ and the ellipse $\omega_{c 1}$.

$l_{\infty}=\left\{P_{\infty}=\left[\begin{array}{llll}x_{w} & y_{w} & 0 & 0\end{array}\right]^{T} \mid A x_{w}+B y_{w}+D * 0=0\right\}(5)$

Coordinates of the points lie on the vanishing line $l_{\infty}$ is infinite, so the coefficients $A$ and $B$ in the equation (5) should satisfy $A=B=0$. Thus, the vanishing line $l_{\infty}$ can be rewritten as $l_{\infty}=\left[\begin{array}{lll}0 & 0 & 1\end{array}\right]^{T}$. Conversely, let $O_{w}=\left[\begin{array}{lll}x_{0} & y_{0} & 1\end{array}\right]^{T}$ be the circle center, the $3 \mathrm{D}$ circle $l=\left[\begin{array}{ccc}1 & 0 & -x_{0} \\ 0 & 1 & -y_{0} \\ -x_{0} & -y_{0} & x_{0}^{2}+y_{0}^{2}-r^{2}\end{array}\right]\left[\begin{array}{l}x \\ y \\ 1\end{array}\right]$

Comparing the equation (5) with equation (6), it can be concluded that the line $l$ is a vanishing line. Since the pole-polar relationship is a projective invariance, the vanishing line can be obtained with the image of a circle and the image of the corresponding circle center.

According to the proposition 1, the vanishing line $l_{\infty}$ can be determined by the concentric circle constraint. According to the description in section 2, it can be known that the perspective ellipse $C$ and the IAC $\omega$ have four
Proof. Let $P_{\infty}=\left[\begin{array}{llll}x_{w} & y_{w} & 0 & 0\end{array}\right]^{T}$ be a vanishing point on the concentric circle's supporting plane, the vanishing line can be expressed as

centered at $\left(x_{0}, y_{0}\right)$ with the radius $r$ is $\left(x-x_{0}\right)^{2}+\left(y-y_{0}\right)^{2}=r^{2}$. Thus, the line determined by a pole-polar relationship between the circle center $O_{w}$ and the 3D circle can be expressed as

intersections in common. Two of them are the ICPs. With the introduction of a concentric circle constraint, ICPs can be determined uniquely from the intersections between the vanishing line $l_{\infty}$ and the ellipse $e_{1}$.

Let $o_{c}$ be the image coordinates of the circle center in the CCS. Apparently, there is a pole-polar relationship between the circle center $O_{c}$ and the vanishing line $l_{\infty}$

$l_{\infty} \square \omega_{c} o_{c}$ 
where $\sqcup$ stands for equality up to scale.

Let $I_{m}$ and $J_{m}$ represent the ICPs, and they can be obtained with known IAC $\omega$ and projection equations of concentric circles. The rotation matrix component can be expressed as [1]:

$$
\begin{aligned}
& r_{1}=s_{1} K^{-1} \operatorname{Re}\left(I_{m}\right), \\
& r_{2}=s_{2} K^{-1} \operatorname{Im}\left(I_{m}\right), \\
& r_{3}=s_{3}\left[K^{-1} \operatorname{Re}\left(I_{m}\right)\right] \times\left[K^{-1} \operatorname{Im}\left(I_{m}\right)\right],
\end{aligned}
$$

where $s_{1}, s_{2}$ and $s_{3}$ are constants. $s_{3}$ can be obtained by $\left\|r_{3}\right\|=1$.
The vanishing line passes through the ICPs, and the projection of circle center is solved via the equation (7). Thus, the translation vector $t$ can be recovered by

$t=s_{4} K^{-1} m_{o}$

where $s_{4}$ is a constant, $m_{o}$ is the image of the concentric circle center.

In the case of coplanar circles, position parameters can be optimized with the additional circle constraint [6]. And the orientation parameters can be achieved in another way $[2,5]$. After recovering the circle's projected center, a unit normal to the circle plane can be computed directly

$$
\left[\begin{array}{l}
R_{12} \\
R_{22} \\
R_{32}
\end{array}\right]= \pm N Q\left[\begin{array}{c}
X_{c} \\
Y_{c} \\
f
\end{array}\right]
$$

where $N[\cdot]$ denotes the normalization into a unit vector, $X_{c}$ and $Y_{c}$ are the image coordinates of the circle's

\subsection{NON-COPLANAR CONCENTRIC CIRCLES}

Similar to the case of coplanar circles, the noncoplanar concentric circles are is common enough in our daily life. And here, one of the two non-coplanar concentric circles will be seen as an additional constraint to differentiate the real pose from the other. The 3D coordinates of the circle center and the normal vector of the supporting plane with a known radius $[7,8]$ can be obtained easily.

Suppose $R$ and rare the radii of non-coplanar concentric circles, the perspective projection of the big circle in the image plane and the origin of the CCS determine a cone. Then what we need to do is to find a plane which intersects the cone and the section is a circle with a radius of $\mathrm{R}$. The pose of the small circle can be solved in a similar way. At this point, the ambiguity problem in pose estimation can be shown to have two planes satisfy that the intersecting curve is a circular feature with the desired diameter.

Concentric circle constraint is employed to remove the false intersecting plane. Thus, two 3D cones are determined by two bases (the images of two concentric projected center, $Q$ denotes the ellipse matrix which is defined in [16] circles) and a common vertex (the center of the camera's lens) in Fig. 4. In order to facilitate the analysis, this study chooses a special projection plane in which the projections of two concentric circles are two lines.

According to the conclusion in [7], this study obtain a standard form of the cone after the transformation of the reference frame with a matrix $P$ :

$$
P^{-1} Q P=P^{-T} Q P=\operatorname{diag}\left(\lambda_{1}, \lambda_{2}, \lambda_{3}\right)
$$

It should be noted that the transformation is a pure rotational matrix. Meanwhile, a new frame of which the axis is aligned with the cone's rotation axis is generated. The projections of the cone and the sectioning planes in the XOZ plane are shown in Fig. 5. The distance in the Euclidean space is a geometric invariance. Thus the segment along the generatrix of the object is invariable in CCS or WCS. The clue is used to exclude the false solution.

Projection of the cone and the sectioning planes on the XOZ plane. $M N$ and $M_{1} N_{1}$ are both projections of the non-coplanar concentric circles and a set of parallel lines. 
So are $C D$ and $C_{1} D_{1} . O_{1}, O_{2}, O_{3}$ and $O_{4}$ are the projections of the circle center. (a) The big circle is in front.

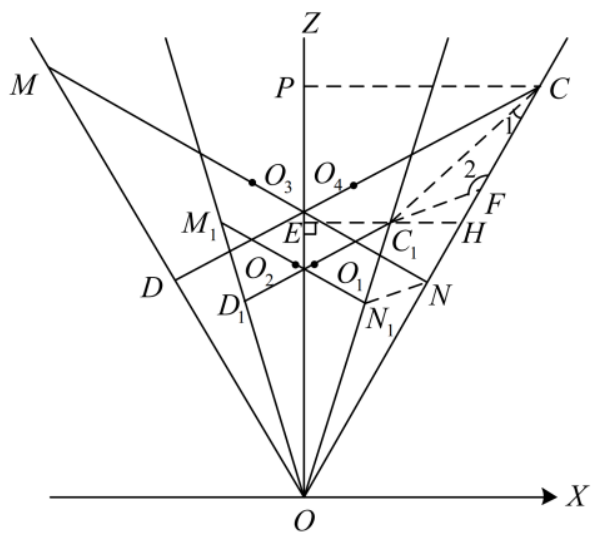

(a) (b) The small is in front.

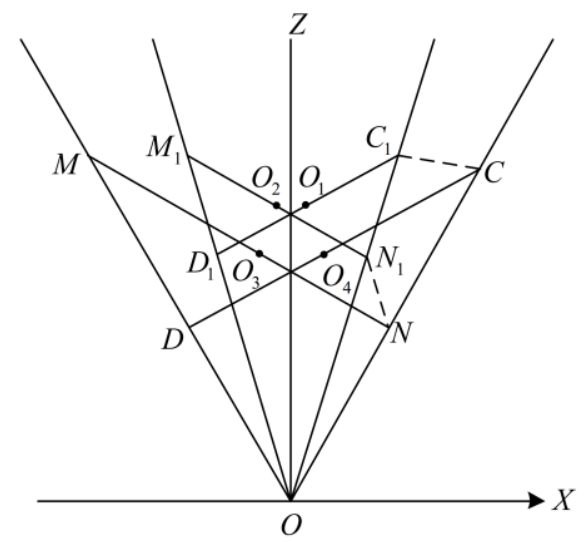

(b)

Figure 5: Projections of the cone and the section planes on the $X O Z$ plane. $M N$ and $M_{l} N_{l}$ are both projections of the noncoplanar concentric circles and a set of parallel lines. So are $C D$ and $C_{1} D_{1} . O_{1}, O_{2}, O_{3}$ and $O_{4}$ are the projections of the circle center. (a) The big circle is closer to the camera. (b) The small is closer to the camera.

$$
\text { If }\left\|C C_{1}\right\| \neq\left\|N N_{1}\right\| \text { can be proved on a condition }
$$

which the distance between the parallel lines is greater than zero and the angle between the line $M N$ and $C D$ is nonzero (that is, there are two possible pose solutions), it can be concluded that the concentric circle constraint is an effective way to remove the false pose.

Proposition 2. The pose parameters can be uniquely determined if $\left\|C C_{1}\right\| \neq\left\|N N_{1}\right\|$ is proved.
Proof. Fig. 5 shows a standard cone and two section planes projected onto the plane $\mathrm{XOZ}$. The dashed lines represent auxiliary lines added for completing the proof. The line $C_{1} F_{1}$ is parallel to line $N N_{1}$, and $C_{1} F_{2}$ is perpendicular to $O Z$. Details of the proof are represented as shown below.

Hence, it can be obtained that

$$
\begin{gathered}
\because N N_{1} \square F C_{1} \\
\therefore \square O N N_{1} \cong \square O F C_{1} \\
\therefore \frac{\left\|N N_{1}\right\|}{\left\|F_{1} C\right\|}=\frac{\|O N\|}{\|O F\|}=\frac{\|O N\|}{\|O N\|+\|N F\|}
\end{gathered}
$$

$$
\left\|F C_{1}\right\|>\left\|N N_{1}\right\|
$$

Let $\angle C_{1} C F=\angle 1, \angle C F C_{1}=\angle 2$

$$
\begin{aligned}
& \because \angle 2=\angle F C_{1} H+\angle C_{1} H F \\
&=\angle F C_{1} H+\angle H E O+\angle H O E \\
&=\angle F C_{1} H+\angle H O E+90^{\circ} \\
& \therefore \angle 2>90^{\circ} \\
& \because \angle 1+\angle P C C_{1}<90^{\circ} \\
& \therefore \angle 2>\angle 1
\end{aligned}
$$


Thus, it can be obtained that

$$
\left\|C C_{1}\right\|>\left\|C_{1} F\right\|>\left\|N_{1} N\right\|
$$

The method for excluding the false pose with the concentric circle constraint is outlined as follows.

- Establishing two ellipse equations as $e_{1}$ and $e_{2}$ with the ellipse fitting algorithm [16].

- Establishing the equation of the cone defined by the base (the projection of the principal circle) and the vertex (the center of the camera's lens).

- Determing $\lambda_{i}(i=1,2,3)$ in the equation (12) with the method proposed in [8].

- Calculating the projections of non-coplanar concentric circles in the plane $X O Z$.

- Computing lengths of the line $C C_{1}$ and $N N_{1}$.

- Removing the false pose solution by comparing the results in step 5 with the real length.

\section{EXPERIMENTAL RESULTS}

In order to verify the method above, the ellipses need to be detected firstly. In this section, the existing ellipse fitting algorithm is directly employed to get the projection equation in tests. Accuracy and efficiency of the pose estimation largely depends on the ellipse-fitting algorithm. Szpak et al.[17] proposed a guaranteed ellipse fitting method that is different from conventional methods[18-20]. They strike a balance between geometric methods and algebraic methods. This guaranteed method is thus employed to achieve ellipse equations in images. Our system setup is shown in Fig.6.

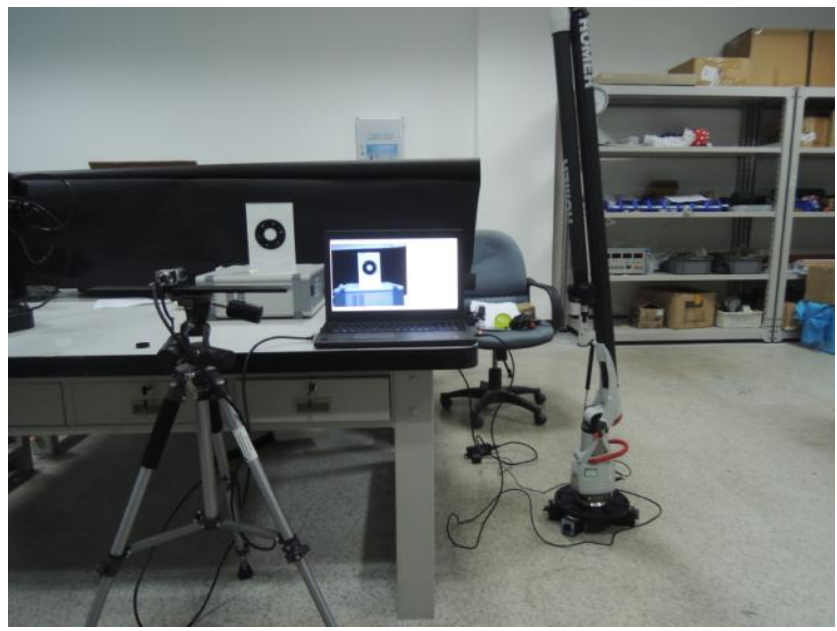

Figure 6: The system setup.

To verify the proposed method, concentric circles of coplanar case and non-coplanar case are tested, respectively. In our experiments, an industrial CCD camera with a resolution of $2456 \times 2058$ is used, and the sensor is pre-calibrated[21]. The matching lens is computer M1224 with a focal length of $12 \mathrm{~mm}$. At the same time, an absolute measuring arm with measurement precision of $0.049 \mathrm{~mm}$ is used to evaluate our method.

Table1:Results from the coplanar case. The calibrated results and measurement results are determined by the absolute measuring arm and the vision system, respectively

\begin{tabular}{|c|c|c|c|c|c|}
\hline \multirow{2}{*}{ Scene } & \multicolumn{2}{|c|}{ Measurement results } & \multicolumn{2}{|c|}{ Calibrated results } & \multirow{2}{*}{ Error(TV) } \\
\cline { 2 - 6 } & $\boldsymbol{U N}$ & $\boldsymbol{T V}$ & $\boldsymbol{U N}$ & $\boldsymbol{T V}$ & \multicolumn{1}{|c|}{} \\
\hline \multirow{3}{*}{$\mathrm{a}$} & {$\left[\begin{array}{c}-0.1065 \\
-0.0859 \\
0.9907\end{array}\right]$} & {$\left[\begin{array}{c}168.1180 \\
-43.8985 \\
698.1721\end{array}\right]$} & {$\left[\begin{array}{c}-0.1097 \\
-0.0832 \\
0.9905\end{array}\right]$} & {$\left[\begin{array}{c}170.1304 \\
-43.6831 \\
701.7931\end{array}\right]$} & {$\left[\begin{array}{l}2.0124 \\
0.2154 \\
3.6210\end{array}\right]$} \\
\hline
\end{tabular}




\begin{tabular}{|c|c|c|c|c|c|c|}
\hline $\mathrm{b}$ & {$\left[\begin{array}{c}0.0127 \\
-0.0768 \\
0.9970\end{array}\right]$} & {$\left[\begin{array}{c}-56.2672 \\
-43.0540 \\
700.0083\end{array}\right]$} & {$\left[\begin{array}{c}0.0159 \\
-0.0755 \\
0.9970\end{array}\right]$} & {$\left[\begin{array}{l}-54.5512 \\
-42.2011 \\
703.9146\end{array}\right]$} & {$\left[\begin{array}{l}1.7160 \\
0.8529 \\
3.9063\end{array}\right]$} \\
\hline $\mathrm{c}$ & {$\left[\begin{array}{c}0.5019 \\
-0.1250 \\
0.8558\end{array}\right]$} & {$\left[\begin{array}{c}-138.5390 \\
-10.0868 \\
746.7406\end{array}\right]$} & {$\left[\begin{array}{c}0.5020 \\
-0.1244 \\
0.8559\end{array}\right]$} & {$\left[\begin{array}{c}-136.4270 \\
-9.1214 \\
749.3097\end{array}\right]$} & {$\left[\begin{array}{l}2.1120 \\
0.9654 \\
2.5691\end{array}\right]$} \\
\hline $\mathrm{d}$ & {$\left[\begin{array}{c}0.4712 \\
-0.1864 \\
0.8621\end{array}\right]$} & {$\left[\begin{array}{c}144.6577 \\
-7.4181 \\
735.9604\end{array}\right]$} & {$\left[\begin{array}{c}0.4729 \\
-0.1839 \\
0.8617\end{array}\right]$} & {$\left[\begin{array}{c}146.3175 \\
-6.9782 \\
738.8505\end{array}\right]$} & {$\left[\begin{array}{l}1.6598 \\
0.4399 \\
2.8901\end{array}\right]$} \\
\hline
\end{tabular}

\subsection{THE COPLANAR CASE}

For the coplanar case, synthetic and real images are tested respectively. The first set of tests utilize the synthetic data and the corresponding pattern is displayed on a computer screen (see Fig.7). A circular part is fixed to a plate in the second set. The experiment results are shown in Table 1 (UN represents the unit normal and TV represents the translation vector).

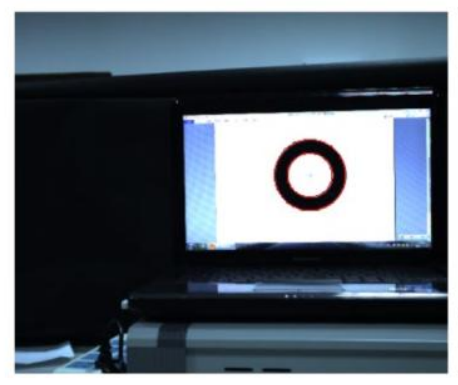

(a)

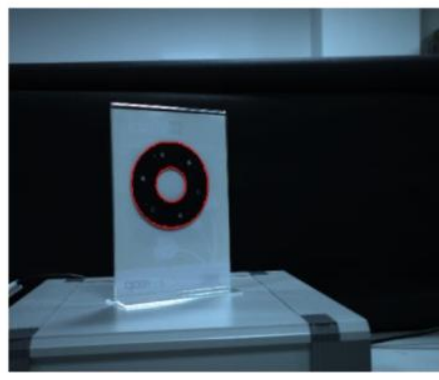

(c)

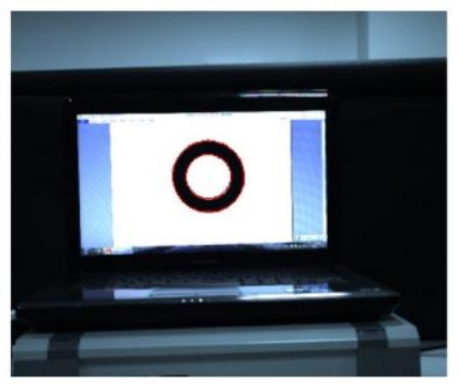

(b)

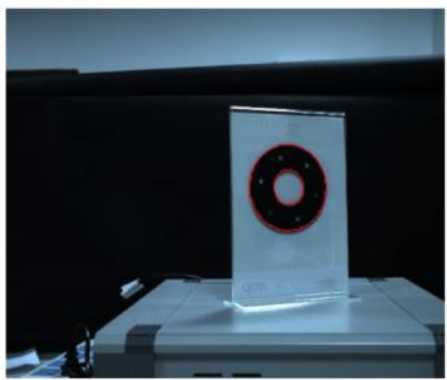

(d)

Figure 7: The coplanar case.(a) and (b) are the first group.(c) and (d) are the second group.

\subsection{THE NON-COPLANAR CASE}

Fig.8 shows two different scenes of the noncoplanar circle feature. The generatrix length between concentric circles (GCC) is $45.21 \mathrm{~mm}$. The real pose of part can be judged according to the GCC. The corresponding experimental results are demonstrated in Table 2 (GCC represents the generatrix length between concentric circles). 


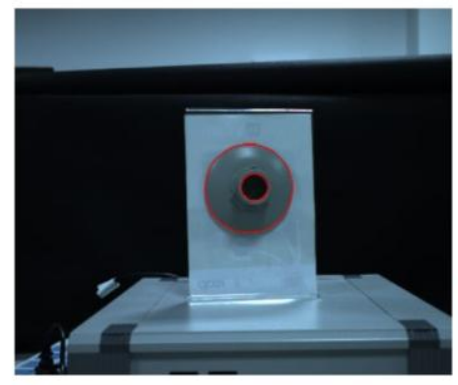

(a)

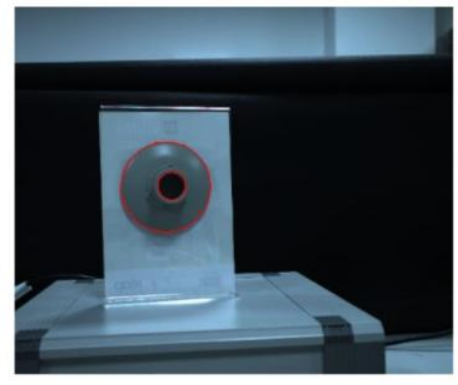

(b)

Figure 8: The non-coplanar case.

Table2:Results from the non-coplanar case. The calibrated results and measurement results are determined by the absolute measuring arm and the vision system, respectively.

\begin{tabular}{|c|c|c|c|c|c|c|}
\hline \multirow{2}{*}{ Scene } & \multicolumn{3}{|c|}{ Measurement results } & \multicolumn{3}{|c|}{ Calibrated results } \\
\hline & $U N$ & $T V$ & $G C C$ & $U N$ & $T V$ & $G C C$ \\
\hline \multirow{6}{*}{$\mathrm{a}$} & 0.2341 & 29.5012 & & \multirow{6}{*}[\begin{array}{c}{0.2370}\\
{-0.1609}\\
{0.9581}\end{array}]{} & \multirow{6}{*}[\begin{array}{c}{30.7920}\\
{-8.9972}\\
{714.3658}\end{array}]{} & \multirow{6}{*}{45.12} \\
\hline & -0.1647 & -8.6430 & 46.98 & & & \\
\hline & 0.9582 & 712.1488 & & & & \\
\hline & {$[-0.2097$} & 25.8187 & & & & \\
\hline & -0.0870 & -7.6664 & 40.24 & & & \\
\hline & 0.9739 & 712.2697 & & & & \\
\hline \multirow{6}{*}{ b } & 0.2201 & -158.0637 & & \multirow{6}{*}[\begin{array}{c}{0.2229}\\
{-0.1521}\\
{0.9629}\end{array}]{} & \multirow{6}{*}[\begin{array}{c}{-159.5591}\\
{-9.5237}\\
{709.5169}\end{array}]{} & \multirow{6}{*}{45.33} \\
\hline & -0.1556 & -9.0703 & 45.70 & & & \\
\hline & 0.9630 & 707.3917 & & & & \\
\hline & {$[-0.2056$} & {$[-154.6250]$} & & & & \\
\hline & -0.0694 & -8.6151 & 41.96 & & & \\
\hline & 0.9762 & 707.5062 & & & & \\
\hline
\end{tabular}

As shown in Table 2, two different GCC can be acquired in each test image because of the pose ambiguity. The correct GCC is closer to the calibrated result than the false GCC corresponding to the ambiguous pose. The pose ambiguity can thus be solved with the aid of GCC. More results in different non-coplanar cases are shown in Fig.9 and Fig. 10. 


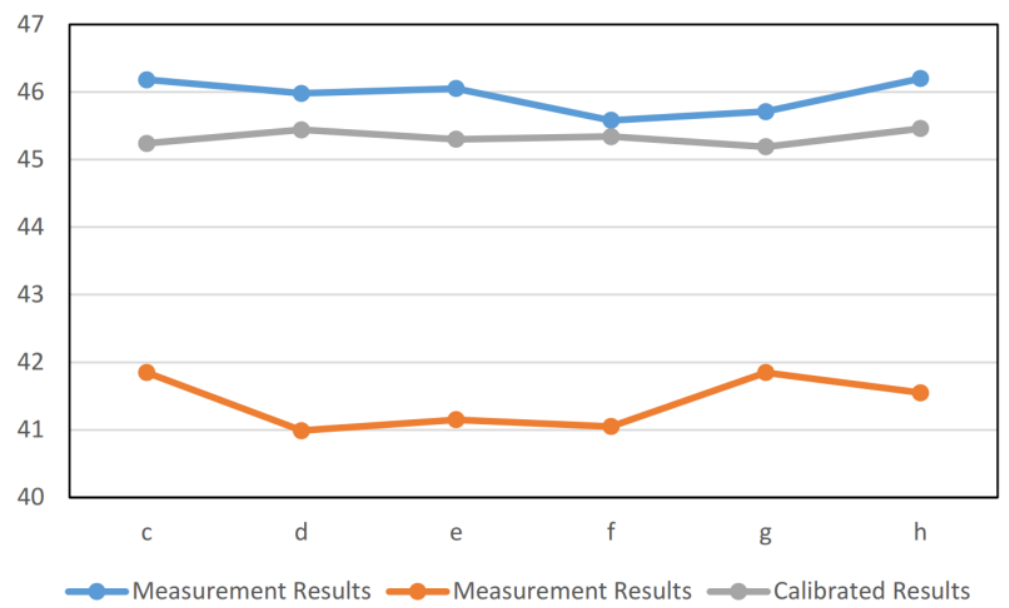

Figure 9: Additional results corresponding to the non-coplanar case.

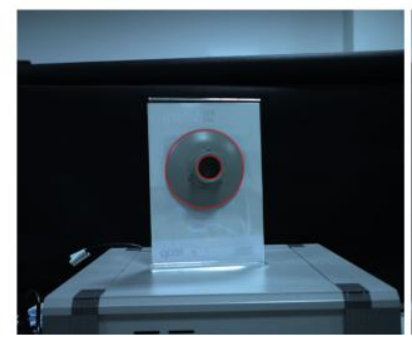

(c)

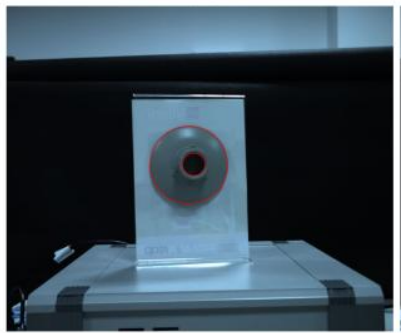

(f)

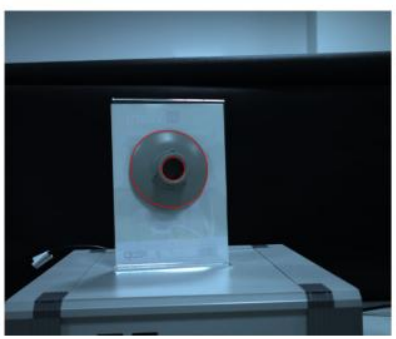

(d)

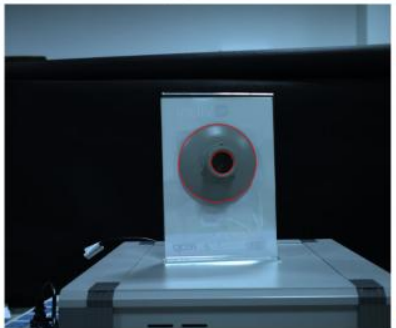

(g)

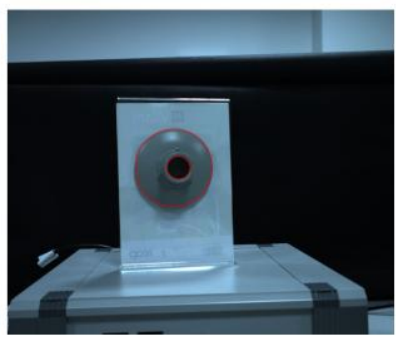

(e)

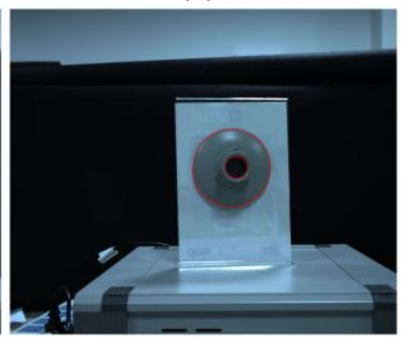

(h)

Figure 10: Additional scenes of the non-coplanar case.

As shown in Fig.9, the deviation between the correct GCC and the calibrated GCC is less than 1 mmover different scenes. Note that the difference between measurement results mainly comes from the manual error.

\section{CONCLUSIONS}

In this paper, this study proposed a new method to exclude the false solution in pose estimation of the circle feature. To this end, a simple concentric circle constraint can be frequently found in real scene is employed. Besides, the proposed method has both simple calculation process and stable results. The results of both synthetic images and real images clearly show the effectiveness of the proposed method. Also, the ellipse detection and ellipse fitting are the main error source for pose estimation with concentric circle feature.

\section{REFERENCES}

[1] Meng, X. \& Hu, Z. (2003). A new easy camera calibration technique based on circular points. Pattern Recognition, 36(5), 1155-1164.

[2] Fremont V. \& Chellali R. (2002). Direct camera calibration using two concentric circles from a single view. In Proceedings of the International Conference on Artificial Reality and Telexistence, Tokyo, Japan, 93-98. 
[3] Abad F., Camahort E., \& Vivó R. (2004). Camera calibration using two concentric circles. In Proceedings of the International Conference Image Analysis and Recognition, Porto, Portugal, 688-696.

[4] Kim J. S., Kim H. W., \& Kweon I. S. (2002). A camera calibration method using concentric circles for vision applications. In Proceedings of the Asian Conference on Computer Vision, Melbourne, Australia. Available at: http://staff.itee.uq.edu.au/lovell/aprs/accv2002/accv2002_pr oceedings/Kim515.pdf.

[5] Jiang G. \& Quan L. (2015 Dec). Detection of concentric circles for camera calibration. In Proceedings of the IEEE International Conference on Computer Vision, Santiago, Chile, 333-340.

[6] Fu S. P., Zhao J. B., Xia R. B., \& Liu W. (2015). Annular mirror based extrinsic camera automatic calibration. Robot, 37, 277-285.

[7] Safaeerad, R., Tchoukanov, I., Smith, K. C., \& Benhabib, B. (1992). Three-dimensional location estimation of circular features for machine vision. IEEE Transactions on Robotics and Automation, 8(5), 624-640.

[8] Shiu, Y. C. \& Ahmad, S. (1989). 3D location of circular and spherical features by monocular model-based vision. systems man and cybernetics. In Proceedings of the IEEE International Conference on Systems, Man and Cybernetics. Available at: https://ieeexplore.ieee.org/abstract/document/71362/citation s\#citations.

[9] Chen, Z. \& Huang, J. (1999). A vision-based method for the circle pose determination with a direct geometric interpretation. IEEE Transactions on Robotics and Automation, 15, 1135-1140.

[10] Zheng, Y., Ma, W., \& Liu, Y. (2008). Another way of looking at monocular circle pose estimation. In Proceedings of the IEEE International Conference on Image Processing, San Diego, USA, 861-864.

[11] Zhang, L., Xu, K.J., Zhao, R., \& Liang, L.P. (2009). Improvement of position and orientation measurement algorithm of monocular vision based on circle features. Journal of Hefei University of Technology, 32, 1669-1673. [12] Wei, Z. Z., Zhao, Z., \& Zhang, G. J. (2010). Solution of duality in pose estimation of single circle using Euclidean angular constraint. Optics and Precision Engineering, 18, 685-691.

[13] Miao, X., Zhu, F., Ding, Q. H., \& Hao, Y. M. (2013). Monocular vision pose measurement based on docking ring component. Acta Optica Sinica, 33, 115-123.

[14] Wang G., Wu J., \& Ji Z. (2008). Single view based pose estimation from circle or parallel lines. Pattern Recognition Letters, 29(7), 977-985.

[15] Fu, M. \& Leng, Y. (2016). A new solution of ambiguity in pose estimation of circle feature using a concentric circle constraint. In Proceedings of the International Conference on Information Science and Technology, Dalian, China, 470-475.

[16] Hartley, R. \& Zisserman, A. (2003). Multiple view geometry in computer vision. Cambridge: Cambridge University Press, 213-220.

[17] Szpak, Z. L., Chojnacki, W., \& Den Hengel, A. V. (2012). Guaranteed ellipse fitting with the sampson distance. In Proceedings of the European Conference on Computer Vision, Florence, Italy, 87-100.

[18] Canny, J. (1986). A computational approach to edge detection. IEEE Transactions on Pattern Analysis and Machine Intelligence, 6, 679-698.

[19] Fitzgibbon, A., Pilu, M., \& Fisher, R. B. (1999). Direct least square fitting of ellipses. IEEE Transactions on Pattern Analysis and Machine Intelligence, 21, 476-480.

[20] Halır, R. \& Flusser, J. (1998). Numerically stable direct least squares fitting of ellipses. In Proceedings of the International Conference in Central Europe on Computer Graphics and Visualization, February, 125-132.

[21] Zhang Z. Y. (2000). A flexible new technique for camera calibration. IEEE Transactions on Pattern Analysis and Machine Intelligence, 22, 1330-1334. 